\title{
Gastrointestinal symptoms in low-dose aspirin users: a comparison between plain and buffered aspirin
}

\author{
J. Jaspers Focks • M. M. Tielemans • L. G. M. van Rossum • \\ T. Eikendal • M. A. Brouwer • J. B. M. J. Jansen • \\ R. J. F. Laheij • F. W. A. Verheugt • M. G. H. van Oijen
}

Published online: 13 February 2014

(C) The Author(s) 2014. This article is published with open access at Springerlink.com

\begin{abstract}
Background Aspirin is associated with gastrointestinal side effects such as gastric ulcers, gastric bleeding and dyspepsia. High-dose effervescent calcium carbasalate (ECC), a buffered formulation of aspirin, is associated with reduced gastric toxicity compared with plain aspirin in healthy volunteers, but at lower cardiovascular doses no beneficial effects were observed.

Aim To compare the prevalence of self-reported gastrointestinal symptoms between low-dose plain aspirin and ECC.

Methods A total of 51,869 questionnaires were sent to a representative sample of the Dutch adult general population
\end{abstract}

Electronic supplementary material The online version of this article (doi:10.1007/s12471-014-0522-3) contains supplementary material, which is available to authorized users.

J. Jaspers Focks $(\bowtie) \cdot$ M. A. Brouwer $\cdot$ F. W. A. Verheugt

Department of Cardiology, Radboud University Nijmegen Medical

Center, 670, Geert Grooteplein Zuid 10, 6525 GA Nijmegen,

the Netherlands

e-mail: J.JaspersFocks@gmail.com

M. M. Tielemans $\cdot$ T. Eikendal

Department of Gastroenterology and Hepatology, Radboud

University Nijmegen Medical Center, Nijmegen, the Netherlands

L. G. M. van Rossum

Department of Health Evidence, Radboud University Nijmegen

Medical Center, Nijmegen, the Netherlands

J. B. M. J. Jansen

Department of Gastroenterology, Elkerliek Hospital, Helmond, the Netherlands

R. J. F. Laheij • M. G. H. van Oijen

Department of Gastroenterology, University Medical Center Utrecht, Utrecht, the Netherlands

M. G. H. van Oijen

Division of Digestive Diseases, David Geffen School of Medicine at UCLA, Los Angeles, CA, USA in December 2008. Questions about demographics, gastrointestinal symptoms in general and specific symptoms, comorbidity, and medication use including bioequivalent doses of ECC $(100 \mathrm{mg})$ and plain aspirin $(80 \mathrm{mg})$ were stated. We investigated the prevalence of self-reported gastrointestinal symptoms on ECC compared with plain aspirin using univariate and multivariate logistic regression analyses.

Results A total of 16,715 questionnaires (32\%) were returned and eligible for analysis. Of these, $911(5 \%)$ respondents reported the use of plain aspirin, 633 (4\%) ECC and 15,171 reported using neither form of aspirin (91\%). The prevalence of self-reported gastrointestinal symptoms in general was higher in respondents using ECC (27.5\%) compared with plain aspirin (26.3\%), but did not differ significantly with either univariate (OR 1.06, $95 \% \mathrm{CI} 0.84-1.33$ ), or multivariate analysis (aOR 1.08, $95 \%$ CI 0.83-1.41). Also, none of the specific types of symptoms differed between the two aspirin formulations.

Conclusions In this large cohort representative of the general Dutch population, low-dose ECC is not associated with a reduction in self-reported gastrointestinal symptoms compared with plain aspirin.

Keywords Low-dose aspirin · Effervescent calcium carbasalate $\cdot$ Gastrointestinal symptoms · Survey ·

Community based population

\section{Introduction}

Optimal antithrombotic therapy has proven to be essential in secondary prevention in cardiovascular disease. In this, aspirin has a pivotal role and is associated with a relative reduction of approximately $25 \%$ in recurrent cardiovascular events [1]. However, gastric toxicity is a well-known side effect of aspirin presenting as gastric or duodenal ulcers, bleeding and 
dyspepsia [1-7]. Of these, dyspepsia is most often reported (in $20-40 \%$ of chronic aspirin users) $[4,7,8]$ and is associated with reduced aspirin compliance $[9,10]$, increased healthcare costs [11] and reduced health-related quality of life [12].

To reduce gastrointestinal damage, different formulations of aspirin have been developed. These formulations either delay the release of aspirin beyond the stomach (entericcoated aspirin), facilitate the transit of aspirin across the gastric mucous layer (PL2200), or increase solubility of aspirin supposedly resulting in lower irritating concentrations on the gastric mucosa (effervescent calcium carbasalate (ECC)). The gastric toxicity of different formulations was mainly studied in high dosages and showed clear benefit over plain aspirin with respect to gastric ulcer formation when studied in healthy volunteers [13-18]. However, when investigating its clinical effect in patients on (low-dose) chronic antiplatelet therapy, no clear beneficial effect on gastrointestinal side effects was noticeable [19-22].

In the Netherlands, a total of 1,290,000 patients use lowdose aspirin of which $41 \%$ are prescribed ECC [23]. No data have been published comparing the effects of ECC and plain aspirin on gastrointestinal symptoms. In this population-based cohort of respondents using low-dose aspirin we studied and compared the prevalence of gastrointestinal symptoms between those using ECC and plain aspirin. We also studied whether respondents using different formulations may report different types of gastrointestinal symptoms.

\section{Methods}

\section{Study population}

We sent 51,869 questionnaires by surface mail to a representative sample of the Dutch population in December 2008. Invited subjects were aged 18 years and above, and randomly selected from municipal databases of five different municipalities selected on their geographical location in the Netherlands, in order to gather a representative sample of the Dutch population. We included returned questionnaires until 31 March 2009. We excluded returned questionnaires with missing elements that were part of the primary outcome measure. We also excluded returned questionnaires in which all baseline characteristics were missing or when the medication was unreadable or if the used aspirin formulation was not reported. The complete cohort has been described previously [24]. The current sample size consisted of those respondents reporting the use of either low-dose plain aspirin or ECC.

The Medical Ethical Committee of the Radboud University Nijmegen assessed the research proposal of this study and concluded that it could be waived for ethical review, as questionnaires were returned and stored anonymously, and (non-)responders would not be contacted again. For this reason, we did not obtain written informed consent.

\section{Questionnaire}

The questionnaire has been used before and was specifically designed for collection of demographic information, gastrointestinal symptoms, and medication use $[25,26]$. Participants were asked whether they suffer from gastrointestinal symptoms in general and about the presence of 26 gastrointestinal symptoms such as nausea, early satiety and bloating. Severity of gastrointestinal symptoms was assessed on a seven-point Likert scale $(0=$ absent, $1=$ almost absent, $2=$ mild, $3=$ moderate, $4=$ moderately severe, $5=$ severe, $6=$ very severe) over the preceding 4 weeks [27]. A symptom was considered to be present if the participants scored $\geq 2$ on the Likert scale.

\section{Outcomes}

Our primary outcome was the presence of gastrointestinal symptoms, which was assessed with the question: 'Do you experience gastrointestinal complaints?' and had to be answered with either 'yes' or 'no'. Secondary outcomes were duration of the primary endpoint and the individual gastrointestinal symptoms among responders who reported the presence of gastrointestinal complaints. The primary and secondary outcomes were compared between respondents reporting the use of low-dose plain aspirin $(80 \mathrm{mg})$ and those using ECC $(100 \mathrm{mg})$.

\section{Statistical analysis}

Statistical analyses were performed with SPSS statistical software, version 16.0 (SPSS, Inc., Chicago, Illinois, USA). Frequency tables were provided describing respondents' baseline characteristics. Pearson's chi squared test was used to compare categorical variables. Continuous variables were compared with the Student $T$-test or the Mann-Whitney U method whenever appropriate. Univariate and multivariate associations for gastrointestinal endpoints in respondents using plain aspirin or ECC were analysed using logistic regression. Two-sided p-values of $<0.05$ were considered statistically significant. Covariates were included in the multivariate analysis if they significantly differed between respondents using ECC versus plain aspirin. In addition, those covariates associated with gastrointestinal symptoms at a level of $p<0.1$ in univariate analysis were included in multivariate analysis. Using forward selection, a covariate was allowed into the multivariate model if it influenced the model with a likelihood ratio significance level of $p<0.05$, and was removed again if its significance level exceeded $p=0.1$ during any of the following steps. The type of formulation used (ECC versus plain aspirin) was forced into the model. 
With respect to the duration of symptoms and the analyses of the individual symptoms, only participants reporting the presence of the primary outcome were selected. The duration of symptoms was compared using the Mann-Whitney U method. The individual symptoms were divided into upper and lower gastrointestinal symptoms and figures were provided describing their frequencies. The sum of the individual symptoms present was categorised according to the number of symptoms present and frequencies were provided.

\section{Results}

A total of 18,317 (35\%) questionnaires were returned, of which 742 unopened for various reasons (Fig. 1). After applying our predetermined exclusion criteria a total of 16,715 questionnaires were included in our analyses. In total, 911 persons (5.4\%) reported plain aspirin use, 633 ECC (3.8 \%) and 15,171 reported not using any form of aspirin (90.8\%). Nearly all baseline characteristics differed between participants with and without aspirin (Supplementary Table 1). When comparing plain aspirin and ECC, participants using ECC were older, reported more comorbidity and were using more concomitant medication (Table 1).

The self-reported prevalence of gastrointestinal symptoms of no aspirin, plain aspirin, and ECC were $25.6 \%, 26.3 \%$, and $27.5 \%$, respectively. We observed no difference between plain aspirin and ECC for self-reported gastrointestinal symptoms (ECC: OR 1.06, $95 \%$ CI 0.84-1.33). Also after adjustment with multivariate regression for multiple possible confounders there was no significant difference between plain

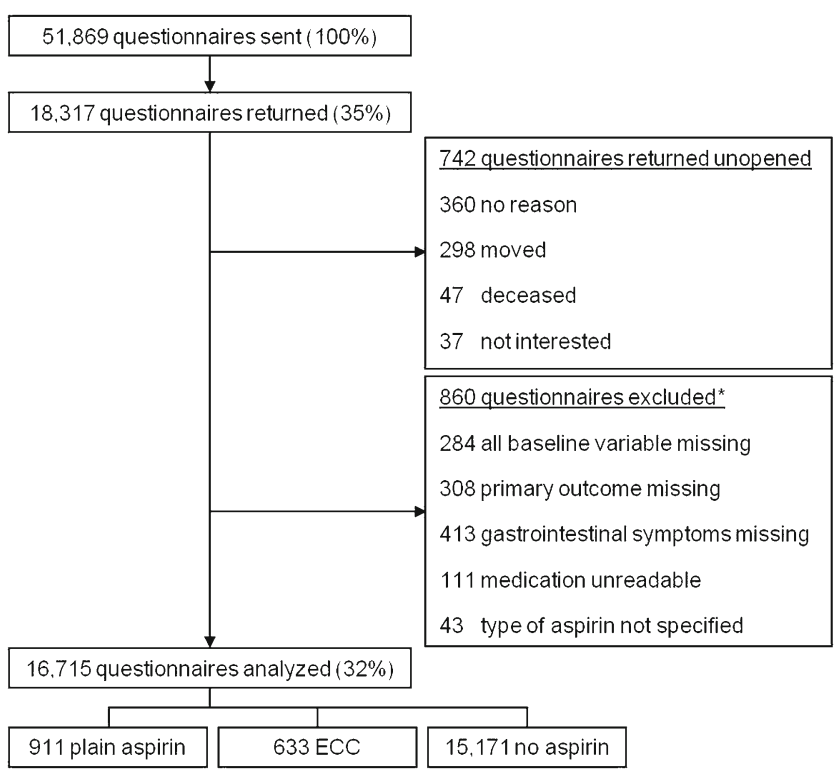

Fig. 1 Flow chart. *Some respondents fulfilled more than 1 exclusion criterion
Table 1 Baseline characteristics

\begin{tabular}{|c|c|c|c|}
\hline & $\begin{array}{l}\text { Plain } \\
\text { aspirin } \\
N=911\end{array}$ & $\begin{array}{l}\text { Effervescent } \\
\text { calcium } \\
\text { carbasalate } \\
N=633\end{array}$ & $P$-value \\
\hline Mean age $( \pm \mathrm{SD})$ (years) & $59.7(15.2)$ & $64.7(11.3)$ & $<0.01$ \\
\hline Male (\%) & $494(56)$ & $377(61)$ & 0.09 \\
\hline Smoking (\%) & $160(18)$ & $116(19)$ & 0.74 \\
\hline $\mathrm{BMI}( \pm \mathrm{SD})\left(\mathrm{kg} / \mathrm{m}^{2}\right)$ & $26.3(4.6)$ & $27.0(4.9)$ & $<0.01$ \\
\hline \multicolumn{4}{|l|}{ Comorbidity (\%) } \\
\hline Diabetes mellitus & $108(12)$ & $106(17)$ & $<0.01$ \\
\hline Rheumatoid arthritis & $53(6)$ & $54(9)$ & 0.04 \\
\hline Asthma/COPD & $62(7)$ & $69(11)$ & $<0.01$ \\
\hline Coeliac disease & $16(2)$ & $9(1)$ & 0.61 \\
\hline IBD & $27(3)$ & $18(3)$ & 0.89 \\
\hline \multicolumn{4}{|l|}{ Medication use (\%) } \\
\hline PPI & $191(21)$ & $188(30)$ & $<0.01$ \\
\hline H2RA & $24(3)$ & $14(2)$ & 0.60 \\
\hline Antacids & $79(9)$ & $50(8)$ & 0.59 \\
\hline Paracetamol & $474(52)$ & $276(44)$ & $<0.01$ \\
\hline NSAIDs & $274(30)$ & $186(29)$ & 0.77 \\
\hline Clopidogrel & $17(2)$ & $36(6)$ & $<0.01$ \\
\hline Dipyridamole & $43(5)$ & $69(11)$ & $<0.01$ \\
\hline Beta blockers & 351 (39) & $301(48)$ & $<0.01$ \\
\hline ACE inhibitors & 175 (19) & $189(30)$ & $<0.01$ \\
\hline Angiotensin-receptor antagonist & $103(11)$ & $83(13)$ & 0.28 \\
\hline Calcium antagonist & $128(14)$ & $105(17)$ & 0.17 \\
\hline Diuretics & $185(20)$ & $155(25)$ & 0.051 \\
\hline Statins & $396(44)$ & $373(59)$ & $<0.01$ \\
\hline Systemic corticosteroids & $15(2)$ & $11(2)$ & 0.89 \\
\hline Oral glucose lowering agents & $85(9)$ & $70(11)$ & 0.27 \\
\hline Antidepressants & $47(5)$ & $40(6)$ & 0.33 \\
\hline \multicolumn{4}{|l|}{ History (\%) } \\
\hline Peptic ulcer disease & $69(8)$ & $76(12)$ & $<0.01$ \\
\hline Peptic ulcer bleeding & $26(3)$ & $15(2)$ & 0.56 \\
\hline
\end{tabular}

$S D$ standard deviation, $B M I$ body mass index, $\mathrm{kg} / \mathrm{m}^{2}=$ kilogram per square meter, $C O P D$ chronic obstructive pulmonary disease, IBD inflammatory bowel disease, $P P I$ proton pump inhibitor, H2RA H2-receptor antagonist, NSAID non-steroid anti-inflammatory disease, $A C E$ angiotensin converting enzyme

aspirin and ECC for the presence of gastrointestinal symptoms (ECC: aOR 1.08, $95 \%$ CI 0.83-1.41, Table 2). Among those reporting gastrointestinal symptoms, respondents using ECC had a significantly longer history of symptoms (10 years, IQR 4-20) compared with participants using plain aspirin (7 years, IQR 3-16, $p=0.04$ ).

In respondents reporting the presence of gastrointestinal symptoms and using either plain aspirin or ECC the presence of no more than one individual upper gastrointestinal symptom was reported by $26.9 \%$ while five or more symptoms 
Table 2 Multivariate logistic regression model for reporting gastrointestinal symptoms with effervescent calcium carbasalate entered into the model

\begin{tabular}{lrrr}
\hline & aOR & $95 \% \mathrm{CI}$ & $P$-value \\
\hline Age (per year increase) & 0.98 & $0.97-0.99$ & $<0.01$ \\
Male gender & 0.71 & $0.55-0.92$ & 0.01 \\
Comorbidity & & & \\
$\quad$ Asthma/COPD & 1.54 & $1.01-2.36$ & 0.046 \\
IBD & 2.01 & $1.00-4.04$ & 0.050 \\
Medication use & & & \\
$\quad$ PPI & 3.96 & $2.96-5.30$ & $<0.01$ \\
$\quad$ H2RA & 4.39 & $2.01-9.57$ & $<0.01$ \\
$\quad$ Antacids & 2.90 & $1.90-4.44$ & $<0.01$ \\
Paracetamol & 1.42 & $1.09-1.86$ & $<0.01$ \\
$\quad$ Effervescent calcium carbasalate & 1.08 & $0.83-1.41$ & 0.57 \\
History & & & \\
$\quad$ Peptic ulcer disease & 2.39 & $1.60-3.58$ & $<0.01$ \\
\hline
\end{tabular}

$a O R$ adjusted odds ratio, $C I$ confidence interval, $C O P D$ chronic obstructive pulmonary disease, $I B D$ inflammatory bowel disease, $P P I$ proton pump inhibitor, $H 2 R A \mathrm{H} 2$-receptor antagonist

were reported present by $32.3 \%$. The most frequently reported upper gastrointestinal symptoms were bloating $(61 \%)$, belching (47\%) and regurgitation (42\%) (Fig. 2a). With respect to lower gastrointestinal symptoms, $23.0 \%$ reported no more than one symptom, while $39.0 \%$ experienced the presence of 5 or more symptoms. Flatulence $(70 \%)$ and borborygmi $(56 \%)$ were the most frequently reported lower gastrointestinal symptoms (Fig. 2b). No significant differences between plain aspirin and ECC were present for any of the upper or lower gastrointestinal symptoms.

\section{Discussion}

We aimed to compare the prevalence of self-reported gastrointestinal symptoms between respondents using plain aspirin and those who were prescribed ECC. We observed that in respondents using any form of low-dose aspirin the prevalence of gastrointestinal symptoms was $27 \%$. The use of ECC is not associated with less gastrointestinal symptoms compared with plain aspirin. The most reported upper gastrointestinal symptoms were bloating, belching, and regurgitation, whereas flatulence and borborygmi were reported most for lower gastrointestinal symptoms. No differences in the type of symptoms between users of ECC and plain aspirin were observed.

The prevalence of gastrointestinal symptoms in our study cohort is in line with previously reported data of aspirin users $[4,7,8]$. Interestingly, the prevalence of gastrointestinal symptoms in our non-aspirin using population is comparable with those who use aspirin. The selection of our study population could have contributed to this finding. Low-dose aspirin is generally a long-term treatment, i.e. for the remainder of the patients' life span. For our study we selected all low-dose aspirin users from a large cohort of randomly selected participants returning the questionnaire. As a result of this study design the odds that aspirin treatment was recently initiated for our participants are minimal. Those patients who suffered from gastrointestinal symptoms during (the initiation of) aspirin treatment were likely to receive co-treatment with a proton pump inhibitor, H2-receptor antagonist or antacid or were even switched to other antiplatelet agents. Consequently, our cohort may consist of a selected population of respondents in whom aspirin is relatively well tolerated. This hypothesis is supported by the more frequent use of gastroprotective agents in low-dose aspirin users compared with our non-aspirin using population (e.g. proton pump inhibitor use: $25 \%$ vs $9 \%$ ). Irrespectively, our data indicate that ECC is of no beneficial value for gastrointestinal symptoms among our population of long-term aspirin users.

So far, only two studies have been conducted to investigate endoscopically proven gastric mucosal damage in users of ECC and plain aspirin. In a randomised cross-over trial,
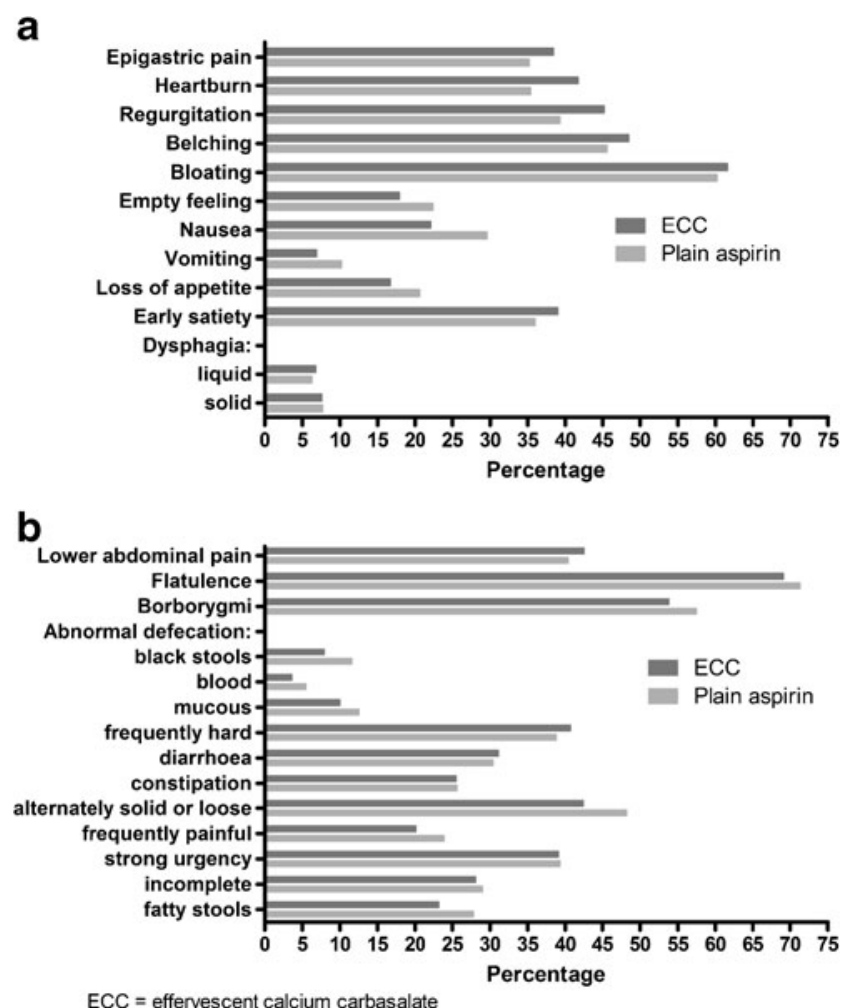

Fig. 2 Type and prevalence of specific upper (a) and lower (b) gastrointestinal symptoms in respondents experiencing gastrointestinal symptoms categorised by aspirin formulation 
ECC significantly reduced endoscopically observed gastric erosions and ulcers compared with the bioequivalent dose of plain aspirin [13]. However, this study assessed healthy volunteers, investigated very high doses of aspirin $(650 \mathrm{mg}$ three times a day) and only studied the short-term effects. More recently, the effects of low-dose ECC and plain aspirin were compared in patients using long-term aspirin for cardiovascular prevention [19]. In this large retrospective cohort study, the authors concluded that the incidence rates of endoscopically proven peptic ulcers were not significantly different between the two groups.

This is the first study comparing the effects of ECC with plain aspirin for gastrointestinal symptoms. Moreover, in order to obtain a representative sample, persons were randomly selected through databases of local authorities without stringent inclusion and exclusion criteria. We do acknowledge some limitations in our study. First, due to our study design, response bias could be a potential limitation. Due to concealment we were unable to contact non-responders and compare their characteristics with responders. To minimise the effect of response bias all participants were invited with a personalised invitational letter and were asked explicitly to participate irrespective of experiencing gastrointestinal symptoms. Seventy-five percent of all respondents indeed did not report the presence of gastrointestinal symptoms. Secondly, we did not study the duration of low-dose aspirin use or the effect of gastrointestinal symptoms on aspirin compliance. Finally, we observed important differences in baseline characteristics between the two aspirin formulations, all to the detriment of those participants using ECC. This could be an indication that physicians are more likely to prescribe plain aspirin to the relatively healthy subjects and preferentially prescribe ECC to the older and more fragile patients. In order to adjust for this possible bias we performed multivariate analysis. Nonetheless, this observation suggesting confounding by indication should be noted and calls for a study with random allocation of aspirin formulation.

We observed that low-dose ECC is not associated with a reduction in gastrointestinal symptoms compared with plain aspirin. This absence of a beneficial effect of ECC over plain aspirin is in analogy to a previous study indicating that lowdose ECC is not associated with a reduction in the prevalence of gastric ulcer complications. Notably, the costs of ECC are significantly higher compared with plain aspirin ( $€ 1.55$ vs. $€ 0.79 /$ month in the Netherlands) [28]. With 530,000 ECC users in the Netherlands, these additive costs comprise nearly 5 million euro annually. In view of the lack of a beneficial clinical effect and the higher costs of ECC, we feel that plain aspirin is the first drug of choice. If gastrointestinal symptoms occur, we advise to prescribe a relatively cheap proton pump inhibitor with proven beneficial effects [29-31]. Only if this does not reduce the symptoms, might one consider ECC as an alternative to plain aspirin.
In conclusion, the prevalence of gastrointestinal symptoms among aspirin users in the Dutch community is $27 \%$ with no difference between effervescent calcium carbasalate and plain aspirin in overall prevalence and type of symptoms reported.

Funding This work was supported by an unrestricted research grant from Nycomed BV, the Netherlands. The sponsor did not have any influence on study design, data collection, analysis or writing of the manuscript.

Conflict of interest J. Jaspers Focks has received speaker fees or honoraria for advisory boards from Bayer Healthcare, Boehringer Ingelheim, Bristol-Meyers Squibb, Daiichi Sankyo and Pfizer. F.W.A. Verheugt has received educational and research grants from Bayer Healthcare, Roche, Eli Lilly and Boehringer Ingelheim and received honoraria for consultancies from Daiichi-Sankyo, Eli Lilly, Merck, The Medicines Company and Bayer Healthcare. Martijn G.H. van Oijen has served as a consultant for AstraZeneca and Pfizer, and has received unrestricted research grants from AstraZeneca, Nycomed, Janssen, and Shire pharmaceuticals. M.M. Tielemans, L.G.M. van Rossum, T. Eikendal, M.A. Brouwer, J.B.M.J. Jansen and R.J.F. Laheij have no conflicts to declare.

Open Access This article is distributed under the terms of the Creative Commons Attribution License which permits any use, distribution, and reproduction in any medium, provided the original author(s) and the source are credited.

\section{References}

1. Antithrombotic Trialists' (ATT) Collaboration, Baigent C, Blackwell $\mathrm{L}$, et al. Aspirin in the primary and secondary prevention of vascular disease: collaborative meta-analysis of individual participant data from randomized trials. Lancet. 2009;373(6978):1849-60.

2. Lanas A, Wu P, Medin J, et al. Low doses of acetylsalicylic acid increase risk of gastrointestinal bleeding in a meta-analysis. Clin Gastroenterol Hepatol. 2011;9(9):762-8.

3. Lanas A, McCarthy D, Voelker M, et al. Short-term acetylsalicylic acid (aspirin) use for pain, fever, or colds - gastrointestinal adverse effects: a meta-analysis of randomized clinical trials. Drugs R D. 2011;11(3):277-88.

4. Yeomans ND, Lanas AI, Talley NJ, et al. Prevalence and incidence of gastroduodenal ulcers during treatment with vascular protective doses of aspirin. Aliment Pharmacol Ther. 2005;22(9):795-801.

5. Niv Y, Battler A, Abuksis G, et al. Endoscopy in asymptomatic minidose aspirin consumers. Dig Dis Sci. 2005;50(1):78-80.

6. Derry S, Loke YK. Risk of gastrointestinal haemorrhage with longterm use of aspirin: meta-analysis. BMJ. 2000;321(7270):1183-7.

7. Laheij RJ, Jansen JB, Verbeek AL, et al. Helicobacter Pylori infection as a risk factor for gastrointestinal symptoms in patients using aspirin to prevent ischaemic heart disease. Aliment Pharmacol Ther. 2001;15(7):1055-9.

8. Pratt S, Thompson VJ, Elkin EP, et al. The impact of upper gastrointestinal symptoms on nonadherence to, and discontinuation of, low-dose acetylsalicylic acid in patients with cardiovascular risk. Am J Cardiovasc Drugs. 2010;10(5):281-8.

9. Scheiman JM, Devereaux PJ, Herlitz J, et al. Prevention of peptic ulcers with esomeprazole in patients at risk of ulcer development treated with low-dose acetylsalicylic acid: a randomized, controlled trial (OBERON). Heart. 2011;97(10):797-802. 
10. Cayla G, Collet JP, Silvain J, et al. Prevalence and clinical impact of upper gastrointestinal symptoms in subjects treated with low dose aspirin: the UGLA survey. Int J Cardiol. 2012;156(1):69-75.

11. De Groot NL, van Haalen HG, Spiegel BM, et al. Gastroprotection in low-dose aspirin users for primary and secondary prevention of ACS: results of a cost-effectiveness analysis including compliance. Cardiovasc Drugs Ther. 2013;27(4):341-57.

12. Aro P, Talley NJ, Agréus L, et al. Functional dyspepsia impairs quality of life in the adult population. Aliment Pharmacol Ther. 2011;33(11):1215-24.

13. Murray FE, Hudson N, Atherton JC, et al. Comparison of effects if calcium carbasalate and aspirin on gastroduodenal mucosal damage in human volunteers. Gut. 1996;38(1):11-4.

14. Petroski D. Endoscopic comparison of three aspirin preparations and placebo. Clin Ther. 1993;15(2):314-20.

15. Hoftiezer JW, Silvoso GR, Burks M, et al. Comparison of the regular and enteric-coated aspirin on gastroduodenal mucosa of man. Lancet. 1980;2(8195):609-12.

16. Cryer B, Bhatt DL, Lanza FL, et al. Low-dose aspirin-induced ulceration is attenuated by aspirin-phosphatidylcholine: a randomized clinical trial. Am J Gastroenterol. 2011;106(2):272-7.

17. Cole AT, Hudson N, Liew LC, et al. Protection of human gastric mucosa against aspirin-enteric coating or dose reduction? Aliment Pharmacol Ther. 1999;13(2):187-93.

18. Dammann HG, Burkhardt F, Wolf N. Enteric coating of aspirin significantly decreases gastroduodenal mucosal lesions. Aliment Pharmacol Ther. 1999;13(8):1109-14.

19. Van Oijen MG, Dieleman JP, Laheij RJ, et al. Peptic ulcerations are related to systemic rather than local effects of low-dose aspirin. Clin Gastroenterol Hepatol. 2008;6(3):309-13.

20. Kelly JP, Kaufman DW, Jurgelon JM, et al. Risk of aspirin-associated major upper-gastrointestinal bleeding with enteric-coated or buffered product. Lancet. 1996;348(9039):1413-16.

21. De Abajo FJ, Garcia Rodriguez LA. Risk of upper gastrointestinal bleeding and perforation associated with low-dose aspirin as plain and enteric-coated formulations. BMC Clin Pharmacol. 2001;1:1.
22. Van Oijen MGH, Jaspers Focks J, Laheij RJF. Comparison of incident dyspepsia between low-dose plain aspirin and enteric-coated aspirin. Clin Gastroenterol Hepatol. 2010;8(4):395.

23. The Netherlands Foundation for Pharmaceutical Statistics/www.s2.nl; Facts and Figures of 2012; accessed on May 21st 2013.

24. Tielemans MM, Jaspers Focks J, van Rossum LGM, et al. Gastrointestinal symptoms are still prevalent and negatively impact health-related quality of life: a large cross-sectional population based study in the Netherlands. PLOS One. 2013;8(7): e69876.

25. Van Marrewijk CJ, Mujakovic S, Fransen GA, et al. Effect and cost-effectiveness of step-up versus step-down treatment with antacids, H2-receptor antagonists, and proton pump inhibitors in patients with new onset dyspepsia (DIAMOND study): a primary-care-based randomised controlled trial. Lancet. 2009;373(9659):215-25.

26. Bovenschen HJ, Janssen MJ, van Oijen MG, et al. Evaluation of a gastrointestinal symptoms questionnaire. Dig Dis Sci. 2006;51(9): 1509-15.

27. Likert R. A technique for the measurement of attitudes. Arch Psychol. 1932;140:55.

28. College voor zorgverzekeringen/www.medicijnkosten.nl; accessed on July 25th 2013.

29. Yeomans ND, Lanas A, Labenz J, et al. Efficacy of esomeprazole (20 mg once daily) for reducing the risk of gastroduodenal ulcers associated with continuous use of low-dose aspirin. Am J Gastroenterol. 2008;103(10):2465-73.

30. Laheij RJ, van Rossum LG, Jansen JB, et al. Proton-pump inhibitor therapy for acetylsalicylic acid associated upper gastrointestinal symptoms: a randomized placebo-controlled trial. Aliment Pharmacol Ther. 2003;18(1):109-15.

31. Scheiman JM, Herlitz J, Veldhuyzen van Zanten SJ, et al. Esomeprazole for prevention and resolution of upper gastrointestinal symptoms in patients treated with low-dose acetylsalicylic acid for cardiovascular protection: the OBERON trial. J Cardiovasc Pharmacol. 2013;61(3):250-7. 